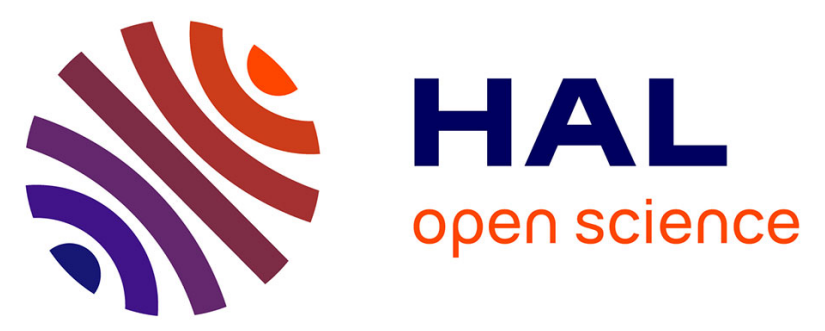

\title{
Loi relative aux libertés et responsabilités des universités (loi LRU), élection du président et conseil d'administration: une analyse en termes de pouvoir
}

Fabrice Barthélémy, Alain Béraud, Mathieu Martin

\section{- To cite this version:}

Fabrice Barthélémy, Alain Béraud, Mathieu Martin. Loi relative aux libertés et responsabilités des universités (loi LRU), élection du président et conseil d'administration: une analyse en termes de pouvoir. Revue d'Economie Politique, 2008, 118 (3), pp.299-315. halshs-00444541

\author{
HAL Id: halshs-00444541 \\ https://shs.hal.science/halshs-00444541
}

Submitted on 6 Jan 2010

HAL is a multi-disciplinary open access archive for the deposit and dissemination of scientific research documents, whether they are published or not. The documents may come from teaching and research institutions in France or abroad, or from public or private research centers.
L'archive ouverte pluridisciplinaire HAL, est destinée au dépôt et à la diffusion de documents scientifiques de niveau recherche, publiés ou non, émanant des établissements d'enseignement et de recherche français ou étrangers, des laboratoires publics ou privés. 


\title{
Loi relative aux libertés et responsabilités des universités (loi LRU), élection du président et conseil d'administration : une analyse en termes de pouvoir
}

\author{
Fabrice BARTHÉLÉMY, Alain BÉRAUD et Mathieu MARTIN \\ THEMA \\ Université de Cergy Pontoise \\ 33 boulevard du Port \\ 95011 Cergy Pontoise Cedex
}

\begin{abstract}
Résumé
L'objet de cet article est de mesurer le pouvoir des membres des conseils d'administration des universités françaises tel qu'il est défini par la loi relative aux libertés et responsabilités des universités (loi LRU). À l'aide d'outils issus de la théorie des jeux coopératifs, et en particulier l'indice de pouvoir de Banzhaf, nous montrons que le nombre de représentants d'un groupe, par exemple les professeurs des universités ou les étudiants, et le pouvoir, sont des notions dont les relations sont parfois surprenantes. Nous présentons des exemples où le réel pouvoir de décision n'appartient pas forcément aux groupes auxquels nous pensions intuitivement.
\end{abstract}

Mots clés : conseil d'administration, indice de Banzhaf, pouvoir, universités françaises.

Classification JEL : C7, D7 
Fabrice Barthélémy :

THEMA, Université de Cergy-Pontoise

33 boulevard du Port

95011 Cergy Pontoise Cedex

Tel. : +33 (0)134256253

Fax : +33 (0)134256233

E-mail : fabrice.barthelemy@u-cergy.fr

Mathieu Martin :

THEMA, Université de Cergy-Pontoise

33 boulevard du Port

95011 Cergy Pontoise Cedex

Tel. : +33 (0)1342562 17

Fax : +33 (0)134256233

E-mail : mathieu.martin@u-cergy.fr

Alain Béraud :

THEMA, Université de Cergy-Pontoise

33 boulevard du Port

95011 Cergy Pontoise Cedex

Tel. : +33 (0)134256175

Fax : +33 (0)134256233

E-mail : alain.beraud@u-cergy.fr 


\title{
The law relative to the liberties and the responsabilities of universities (the "LRU" law), election of the president and board of directors: an analysis in terms of power
}

\begin{abstract}
The aim of this article is to measure the power of the members of french universities boards of directors, power such as mentioned by the law relative to the liberties and the responsabilities of universities (in french, the "LRU" law). We use tools from the cooperative game theory, and particularly the power index of Banzhaf. We show that the number of the representatives of a given group, for instance the professors or the students, and the power, are concepts the relationships of which are sometimes surprising. We provide examples where the real power of decision does not necessarily belong to the groups we thought of intuitively.
\end{abstract}

Keywords: board of directors, Banzhaf power index, power, french universities.

JEL classification: $\mathrm{C} 7, \mathrm{D} 7$ 


\section{Introduction}

Le moins que l'on puisse dire est que la loi relative aux libertés et responsabilités des universités (loi LRU) a suscité bien des controverses. Certains affirment qu'il s'agit de la réforme conduisant à l'autonomie des universités tant attendue, alors que ses détracteurs voient en cette loi la privatisation annoncée de l'enseignement supérieur français et un pouvoir démesuré qu'elle accorde aux présidents des universités et aux conseils d'administration.

L'objet de cet article n'est pas d'alimenter la polémique, mais d'étudier à l'aide d'outils théoriques le pouvoir des différents groupes formant le conseil d'administration. Cette question semble en effet primordiale, même si d'autres aspects non étudiés ici, sont au moins aussi importants. La loi stipule que le nombre d'étudiants dans un conseil d'administration est compris entre 3 et 5 alors que le nombre d'enseignants est compris entre 8 et 14 . Peut-on en conclure que le pouvoir des enseignants peut être jusqu'à 5 fois supérieur? La théorie des jeux coopératifs nous permet de faire une analyse plus fine et de montrer que le lien entre nombre de représentants et pouvoir est parfois surprenant, car non intuitif.

L'exemple souvent cité dans la littérature est celui d'un conseil d'administration à trois actionnaires, où l'un possède $50 \%$ des voix, le second $49 \%$ des voix et le dernier $1 \%$ des voix, les décisions se prenant à la majorité. Le premier actionnaire, pour obtenir la majorité, a besoin d'un autre actionnaire, quel qu'il soit, le but n'étant pas d'obtenir une large majorité, mais simplement plus de $50 \%$ des voix. De ce point de vue, le deuxième et le troisième actionnaire ont le même pouvoir, c'est-à-dire la même capacité d'empêcher ou non la constitution d'une majorité. Bien entendu, l'actionnaire ayant le plus de pouvoir est le premier, car toute majorité est impossible sans son accord. Mais le point contre-intuitif demeure le fait que le second actionnaire, avec un nombre de voix 49 fois supérieur, a le même pouvoir que le troisième!

Plusieurs manières de calculer le pouvoir existent dans la théorie des jeux coopératifs, mais toutes ont pour point commun de mettre en avant la notion de joueur pivot. Il s'agit d'un joueur, qui, par le simple fait de quitter une coalition gagnante, c'est-à-dire majoritaire, rend celle-ci perdante, ou minoritaire. Parmi les différents indices de pouvoir, nous utilisons l'indice de Banzhaf, pour des raisons historiques et normatives. Pour calculer l'indice de Banzhaf, on considère les $2^{n}-1$ coalitions non vides possibles. La probabilité qu'un joueur soit pivot s'obtient en divisant le nombre de fois où ce joueur est pivot par le nombre de 
coalitions possibles $\left(2^{n}-1\right)$. On obtient ainsi l'indice de Banzhaf non normalisé. L'indice de Banzhaf normalisé pour un joueur donné est la part relative de la probabilité d'être pivot pour ce joueur, dans la somme totale de ces probabilités sur tous les joueurs. La version normalisée est celle utilisée tout au long de l'article. Notons qu'elle peut se ramener à une interprétation plus intuitive. Dans l'exemple des actionnaires, toutes les coalitions sont minoritaires exceptées les trois coalitions constituées : du premier et du second actionnaires, du premier et du troisième, des trois actionnaires. Dans les deux premiers cas, coalitions à deux joueurs, les deux actionnaires sont pivots, car si un des deux quitte la coalition gagnante, la coalition restante (un seul joueur), n'est plus majoritaire. Dans le cas à trois actionnaires, seul le premier est pivot. En effet, si la premier quitte la coalition, les actionnaires deux et trois n'obtiennent pas la majorité. Par contre, si le troisième quitte la coalition, le premier et le deuxième forment une coalition majoritaire. Il en est de même dans le cas où le joueur deux sort de la coalition, la coalition formée du premier et du troisième est majoritaire. Ainsi, dans le cas de la coalition à trois actionnaires, le départ du deuxième ou du troisième n'en change pas le caractère majoritaire. On dénombre alors 5 pivots : le premier actionnaire est pivot dans les trois coalitions gagnantes énoncées précédemment, les deux autres seulement une fois (dans les coalitions à 2 joueurs où ils sont associés au premier actionnaire). Le pouvoir du premier actionnaire est ainsi de $60 \%$, celui du second, comme celui du troisième, étant de $20 \%$.

La loi LRU stipule la taille et la constitution des nouveaux conseils d'administration dans les universités françaises. La taille du conseil est comprise entre 20 et 30 représentants, à laquelle il faut ajouter un membre supplémentaire, lorsque le président est élu hors conseil d'administration. Quatre grands groupes siègent au conseil d'administration, dont le nom synthétique est mentionnée entre paranthèses :

a) Les personnels ingénieurs, administratifs, techniques et des bibliothèques (IATOS),

b) Les étudiants et personnes bénéficiant de la formation continue (étudiants),

c) Les personnalités extérieures (extérieurs), qui se décomposent en trois groupes d'après la loi :

- Les chefs d'entreprises ou cadres dirigeants d'entreprise (extérieurs 1),

- Les autres acteurs du monde économique (extérieurs 1), 
- Les représentants des collectivités territoriales ou de leurs groupements, dont un du conseil régional (extérieurs 2),

d) Les enseignants-chercheurs ou personnels assimilés, les enseignants et les chercheurs (enseignants), qui se décomposent en deux groupes d'après la loi :

- Les Professeurs des universités ou assimilés (professeurs),

- Autres personnels enseignants et/ou chercheurs (MCF).

Le nom synthétique extérieurs 1 est le même pour les chefs d'entreprises et les autres acteurs économiques car ces administrateurs qui sont nommés par le président de l'université, forment certainement un groupe homogène. En revanche, les représentants des collectivités locales sont désignés par celles-ci et forment un groupe à part de ce point de vue. Nous obtenons alors 6 groupes siégeant au conseil d'administration et leur nombre de représentants sont présentés dans le tableau 1. Notons que la loi impose aux groupes professeurs et $M C F$ d'avoir le même nombre de représentants, le nombre d'enseignants, lorsque le président est élu parmi les membres du conseil d'administration, doit donc être pair.

Tableau 1 - Nombre de représentants au conseil d'administration

\begin{tabular}{|l|c|}
\hline $\begin{array}{l}\text { Groupes siégeant au } \\
\text { conseil d'administration }\end{array}$ & $\begin{array}{c}\text { Nombre de } \\
\text { représentants }\end{array}$ \\
\hline IATOS & 2 ou 3 \\
Étudiants & de 3 à 5 \\
Extérieurs & 7 ou 8 \\
Extérieurs 1 & de 4 à 6 \\
Extérieurs 2 & 2 ou 3 \\
Enseignants & de 8 à 14 \\
Professeurs & de 4 à 7 \\
MCF & de 4 à 7 \\
\hline
\end{tabular}

Pour rendre l'analyse cohérente, il faut identifier un groupe, par exemple les étudiants, à un unique joueur ayant autant de voix que le nombre de représentants du groupe considéré. Nous supposons donc que les représentants d'un même groupe votent de la même façon. Autrement dit, nous analysons le pouvoir des groupes tout en sachant que leurs membres pourront défendre des positions différentes. De la même manière, 
nous supposons que les groupes votent de façon indépendante les uns des autres.

Après cette introduction, dans une seconde section, nous présentons les rudiments de la théorie des jeux coopératifs et l'indice de Banzhaf nécessaire à notre étude, l'objectif étant de limiter au maximum l'apport technique tout en expliquant la différence entre voix et pouvoir. La troisième section permet d'étudier l'élection du président, sachant que seulement 4 groupes participent au vote. En effet, les membres extérieurs ne participent pas à l'élection du président. Dans la quatrième section, nous présentons les différentes distributions de pouvoir dans le conseil d'administration dans son ensemble. Six groupes sont alors retenus. Nous distinguons deux cas selon que le président élu appartient ou non au conseil d'administration. Cela revient à analyser s'il fait partie ou non de la liste des professeurs élus ${ }^{1}$, ou s'il est élu hors conseil d'administration. Dans ce cas, la loi stipule d'ajouter un membre au conseil d'administration, ce dernier pouvant comporter alors 31 membres.

\section{La mesure du pouvoir en théorie des jeux coopératifs}

La théorie des jeux coopératifs propose un outil intéressant afin de mesurer le pouvoir d'un joueur, l'indice de pouvoir. La littérature propose de nombreuses façons de mesurer le pouvoir, l'objet n'est pas ici de les présenter tous. Il nous a fallu cependant faire un choix parmi ces différents indices, et c'est assez naturellement que notre attention s'est portée sur l'indice de Banzhaf (1965). En effet, hormis son importance historique indéniable, l'indice de Banzhaf possède de bonnes propriétés d'un point de vue normatif et son calcul est certainement le plus simple et le plus intuitif. Cependant, il est assez aisé de vérifier que des résultats quasiment identiques sont obtenus en utilisant l'indice de Shapley-Shubik (1954), qui est l'autre indice majeur utilisé dans la littérature des indices de pouvoir. Une présentation claire de ces deux indices est donnée par Straffin (1994).

Le pouvoir d'un joueur se calcule dans le cadre d'un jeu de vote, qui est un couple $(N, W)$ où $N$ est l'ensemble des joueurs (avec $|N|=n$ où la notation $|A|$ signifie le nombre d'éléments dans l'ensemble $A$ ) et $W$ l'ensemble des coalitions gagnantes,

\footnotetext{
${ }^{1}$ Nous supposons dans l'article que le président est professeur des universités, ceci étant vrai dans la quasi-totalité des cas. Notre analyse sera cependant aisément transposable au cas où le président fait partie du groupe $M C F$.
} 
c'est-à-dire l'ensemble des groupes de joueurs capables d'imposer leur décision au niveau collectif. L'analyse du pouvoir dans un conseil d'administration peut se faire à l'aide d'un jeu de majorité. Ce dernier s'écrit $\left[w_{1}, \ldots, w_{n}\right]$, où $w_{i}$ est le poids attribué au joueur $i$ ( $w_{i}$ est un nombre entier). Une coalition $S$ est gagnante si la somme des poids des membres qui la composent est plus grande ou égale à la majorité, c'est-à-dire

$$
S \in W \Longleftrightarrow \sum_{i \in S} w_{i}>\bar{w} / 2
$$

avec $\bar{w}=\sum_{i} w_{i}$ et le président n'appartient pas à la coalition $S$, ou

$$
S \in W \Longleftrightarrow \sum_{i \in S} w_{i} \geq \bar{w} / 2
$$

dans le cas où président appartenant à la coalition $S$. La loi stipule en effet qu'en cas de partage égal des voix, celle du président est prépondérante. Dans cet article, en fonction du problème analysé, le nombre de joueurs sera égal à 4 ou 6. L'élection du président se fait avec 4 groupes, le groupe extérieurs ne votant pas. Le conseil d'administration au complet comprend 6 groupes, puisqu'aux groupes précédents s'ajoute le groupe extérieurs, partagé en deux sous-groupes, que nous avons appelés extérieurs 1 et extérieurs 2. Les nombres de représentants par groupe ont été présentés dans le tableau 1.

Bien entendu, en assimilant le nombre de représentants d'un même groupe au poids $\left(w_{i}\right)$ d'un seul joueur, nous supposons que le groupe est homogène et donc que ses représentants votent de la même façon. Cette démarche est adéquate dans une analyse de groupes, c'est-à-dire une analyse a priori, mais devrait être affinée, université par université, pour obtenir une analyse encore plus précise du pouvoir, a posteriori.

Le jeu de majorité que nous venons de présenter peut donc se résumer, en $\left[w_{1}, w_{2}, w_{3}, w_{4}\right]$, pour l'élection du président, ou, en $\left[w_{1}, w_{2}, w_{3}, w_{4}, w_{5}, w_{6}\right]$, pour le conseil d'administration, avec dans chacun des cas, des contraintes sur chaque $w_{i}$ ainsi que sur leur somme.

Une fois le jeu défini, la théorie des jeux coopératifs nous enseigne que le pouvoir d'un joueur se mesure en fonction de sa capacité à rendre une coalition gagnante. En effet, si par son unique départ, un joueur rend une coalition perdante alors qu'elle était gagnante, cela signifie que sa présence est indispensable pour que la coalition impose ses décisions. On comprend aisément que ce joueur a du pouvoir. Si l'on note 
$v(S)=1$, le fait que la coalition $S$ est gagnante (on attribue une valeur $v$ de 1 à la coalition $S$ ) et $v(S)=0$, le fait qu'une coalition est perdante, on dit qu'un joueur $i$ est pivot lorsque $v(S)=1$ et $v(S \backslash\{i\})=0$. L'ensemble $S \backslash\{i\}$ est constitué de tous les individus de $S$ auquel on ôte $i$.

À partir de là, l'indice de Banzhaf se calcule simplement. Il suffit d'écrire toutes les coalitions non-vides possibles (soit $2^{n}-1$ ) et de repérer dans chacune d'elles quels sont les joueurs pivots. La probabilité qu'un joueur soit pivot s'obtient alors en divisant le nombre de fois où ce joueur est pivot par le nombre de coalitions possibles $\left(2^{n}-1\right)$. Ceci correspond à l'indice de Banzhaf non normalisé. L'indice de Banzhaf normalisé pour un joueur donné s'obtient en divisant la probabilité que ce joueur soit pivot, par la somme totale de ces probabilités sur tous les joueurs. Cette approche probabiliste est équivalente à celle, plus intuitive, présentée par Straffin (1994), où l'indice de Banzhaf est le nombre de fois où un joueur est pivot divisé par le nombre total de fois où les joueurs sont pivots. Considérons l'exemple simple suivant à trois joueurs $N=\{1,2,3\}$ où le jeu de vote est $[5,4,1]$. Les sept coalitions que l'on peut envisager sont $\{1\},\{2\},\{3\},\{\mathbf{1}, \mathbf{2}\},\{\mathbf{1}, \mathbf{3}\},\{2,3\},\{\mathbf{1}, 2,3\}$. Les joueurs en gras sont pivots, sachant que la majorité est obtenue avec une somme de poids supérieure à 5 (inégalité stricte) et qu'il n'y a pas de voix prépondérante. Le pouvoir du joueur 1 est égal à $3 / 5$, celui du joueur 2 à $1 / 5$, tout comme celui du joueur 3 . Formellement, on écrit l'indice de Banzhaf du joueur $i$ de la manière suivante,

$$
\beta_{i}=\frac{\sum_{S \subseteq N}[v(S)-v(S \backslash\{i\})]}{\sum_{j \in N} \sum_{S \subseteq N}[v(S)-v(S \backslash\{j\})]}
$$

\section{L'élection du président}

Le président de l'université est élu à la majorité absolue par les membres du conseil d'administration. Seuls les groupes professeurs, MCF, étudiants et IATOS votent pour lui. Le fait que les extérieurs ne votent pas change radicalement le jeu, puisque le conseil d'administration choisissant le président comprend désormais de 13 à 22 membres. La majorité est alors obtenue pour un nombre de membres compris entre 7 et 12 , sans qu'il n'y ait de voix prépondérante. 
Tableau 2 - Élection du président

\begin{tabular}{|cccc|c||cccc|}
\hline \multicolumn{3}{|c|}{ Répartition de sièges } & \multicolumn{1}{|c||}{} & \multicolumn{4}{c|}{ Pouvoir } \\
Pr. & MCF & Etu. & IATOS & $\bar{w}$ & Pr. & MCF & Etu. & IATOS \\
\hline 4 & 4 & 3 & 2 & 13 & 33.33 & 33.33 & 33.33 & 0 \\
4 & 4 & 4 & 2 & 14 & 33.33 & 33.33 & 33.33 & 0 \\
4 & 4 & 3 & 3 & 14 & 33.33 & 33.33 & 16.67 & 16.67 \\
5 & 5 & 3 & 2 & 15 & 33.33 & 33.33 & 33.33 & 0 \\
$\mathbf{4}$ & $\mathbf{4}$ & $\mathbf{5}$ & $\mathbf{2}$ & $\mathbf{1 5}$ & $\mathbf{3 3 . 3 3}$ & $\mathbf{3 3 . 3 3}$ & $\mathbf{3 3 . 3 3}$ & $\mathbf{0}$ \\
4 & 4 & 4 & 3 & 15 & 33.33 & 33.33 & 33.33 & 0 \\
5 & 5 & 4 & 2 & 16 & 33.33 & 33.33 & 33.33 & 0 \\
5 & 5 & 3 & 3 & 16 & 33.33 & 33.33 & 16.67 & 16.67 \\
4 & 4 & 5 & 3 & 16 & 25 & 25 & 41.67 & 8.33 \\
6 & 6 & 3 & 2 & 17 & 33.33 & 33.33 & 33.33 & 0 \\
5 & 5 & 5 & 2 & 17 & 33.33 & 33.33 & 33.33 & 0 \\
5 & 5 & 4 & 3 & 17 & 33.33 & 33.33 & 33.33 & 0 \\
6 & 6 & 4 & 2 & 18 & 33.33 & 33.33 & 33.33 & 0 \\
6 & 6 & 3 & 3 & 18 & 33.33 & 33.33 & 16.67 & 16.67 \\
5 & 5 & 5 & 3 & 18 & 33.33 & 33.33 & 33.33 & 0 \\
$\mathbf{7}$ & $\mathbf{7}$ & $\mathbf{3}$ & $\mathbf{2}$ & $\mathbf{1 9}$ & $\mathbf{3 3 . 3 3}$ & $\mathbf{3 3 . 3 3}$ & $\mathbf{3 3 . 3 3}$ & $\mathbf{0}$ \\
6 & 6 & 5 & 2 & 19 & 33.33 & 33.33 & 33.33 & 0 \\
6 & 6 & 4 & 3 & 19 & 33.33 & 33.33 & 33.33 & 0 \\
7 & 7 & 4 & 2 & 20 & 33.33 & 33.33 & 33.33 & 0 \\
7 & 7 & 3 & 3 & 20 & 33.33 & 33.33 & 16.67 & 16.67 \\
6 & 6 & 5 & 3 & 20 & 33.33 & 33.33 & 33.33 & 0 \\
7 & 7 & 5 & 2 & 21 & 33.33 & 33.33 & 33.33 & 0 \\
7 & 7 & 4 & 3 & 21 & 33.33 & 33.33 & 33.33 & 0 \\
7 & 7 & 5 & 3 & 22 & 33.33 & 33.33 & 33.33 & 0 \\
\hline
\end{tabular}

La loi stipule que le groupe enseignants doit être composée pour moitié de professeurs, le nombre de membres du groupe enseignants doit donc être pair. Les pouvoirs des différents groupes sont présentés dans le tableau 2. Les résultats sont assez tranchés. Dans 19 cas sur 24, les trois groupes professeurs, $M C F$ et étudiants ont le même pouvoir, $33,33 \%$. Seule la répartition $[4,4,5,3]$ permet aux étudiants d'avoir le plus de pouvoir. Les IATOS sont les moins influents dans l'élection du président puisqu'ils n'ont aucun pouvoir dans 19 situations sur 24. Dans le meilleur des cas, ils ont 16,67\% $\mathrm{du}$ pouvoir. Cette situation peut sembler inique puisqu'il est des situations où les étudiants n'ont qu'un représentant de plus que les IATOS, un de moins que les professeurs et ont cependant 33,33\% du pouvoir et les IATOS 0\%. Plus étonnant encore est la répartition de sièges $[7,7,3,2]$ où avec seulement un membre de moins que les étudiants, le groupe IATOS possède un pouvoir nul. Ces derniers, avec 4 membres de moins que chaque groupe d'enseignants possède le même pouvoir qu'eux, puisque la répartition de pouvoir est $(33,33 \%, 33,33 \%, 33,33 \%, 0)$. Plusieurs cas simi- 
laires sont présents dans le tableau 2. Même si le groupe IATOS négocie pour obtenir le nombre maximum de membres qu'il peut avoir, c'est-à-dire 3, cela ne l'empêche pas d'avoir un pouvoir nul. Dans ce cas-là, la répartition des autres membres a de l'importance. Par exemple, le jeu $[4,4,4,3]$ donne une distribution de pouvoir $(33,33 \%, 33,33 \%, 33,33 \%, 0)$, alors que le groupe IATOS aurait plus de pouvoir si le groupe étudiants avait un représentant de plus. Il passerait à $8,33 \%$ de pouvoir.

\section{Le conseil d'administration}

Le conseil d'administration détermine la politique de l'établissement. Les pouvoirs des différents groupes au sein de ce conseil semblent donc d'un intérêt vital. À la différence de la section précédente, nous intégrons le groupe extérieurs, constitué des sept ou huit membres. Au moins un des membres de ce groupe doit être un chef d'entreprise ou un cadre dirigeant d'entreprise, au moins un autre un acteur du monde économique et social. Les collectivités territoriales ou leurs groupements sont représentés par deux ou trois administrateurs, dont un du conseil régional. Ces administrateurs sont désignés par les collectivités concernées. Puisque certains administrateurs sont choisis par le président et d'autres désignés par les collectivités locales, il nous semble judicieux de créer deux groupes, nommés extérieurs 1 et extérieurs 2.

La loi stipule que le nombre de membres du conseil d'administration est augmenté d'une unité lorsque le président est choisi hors de ce conseil. Mais comme il peut déjà en faire partie, le nombre de membres du conseil d'administration peut varier finalement de 20 à 31 . Il faut reconnaître que cela complique sensiblement l'analyse car le nombre de cas augmente largement. En effet, en cas d'égalité, le président possède une voix prépondérante, ce qui signifie qu'une coalition dans laquelle il se trouve peut être gagnante sans avoir la majorité absolue. Pour simplifier quelque peu, nous supposerons que le président est toujours un professeur des universités. Cette hypothèse peut sembler gênante mais elle reflète la réalité dans une très grande majorité des cas. Bien entendu, la même analyse serait possible si le président était un maître de conférences. Au final, le nombre de professeurs peut être égal au nombre des $M C F$ plus 1 . 


\subsection{Le président est dans le conseil}

Les résultats sont reportés dans le tableau 3 présenté en annexe. Plusieurs cas peuvent être notés. Tout d'abord, lorsque les groupes négocient le nombre de représentants, ils doivent avoir conscience du fait que les seuls critères du nombre ou du rapport nombre de représentants sur nombre total de sièges, ne sont pas suffisants, la répartition joue un rôle essentiel. Si nous comparons les répartitions $[6,6,4,2,5,2$,$] et [7,7,3,3,4,3]$, les IATOS ont un représentant de plus dans la seconde (3 contre 2$)$ et un poids relatif supérieur (3/27 contre 2/25). Pourtant, les IATOS ont un pouvoir plus faible dans la seconde répartition $(7,14 \%$ contre $10 \%)$. Mais la négociation des représentants se compliquent dans certains cas. Prenons le jeu $[4,4,5,2,4,3]$, les IATOS ont un pouvoir égal à $3,33 \%$. S'ils estiment que l'écart de représentants avec les étudiants est trop important, ils peuvent négocier de deux manières :

- pour obtenir un siège de plus, et dans ce cas ils ont $10 \%$ du pouvoir,

- pour que les étudiants aient un siège de moins, et dans ce cas ils ont un pouvoir nul!

La prépondérance du vote du président joue bien entendu un rôle important, il suffit par exemple d'étudier la répartition $[4,4,4,2,5,3]$ où les professeurs ont un pouvoir de $23,33 \%$ alors que les $M C F$, avec le même nombre de représentants, n'ont que $16,67 \%$ de pouvoir. Le tableau 3 peut être transformé de manière à obtenir la répartition du pouvoir, pour chacun des groupes (tableau 4). Les 20 valeurs différentes du pouvoir sont présentées de manière décroissante dans la première colonne de ce tableau. En caractère gras figure le mode pour chaque groupe.

Si les professeurs et les MCF ont les pouvoirs les plus élevés, les IATOS et les extérieurs 2 ont les pouvoirs les plus faibles. Les résultats concernant les professeurs et les $M C F$ ne diffèrent qu'en raison de la prépondérance du vote du président et les résultats seraient opposés si le président était un $M C F$. Les étudiants sont dans une situation intermédiaire, quasi-bimodale, et sont clairement mieux représentés que les IATOS. Seulement 10 situations impliquent que les étudiants ont moins de $10 \%$ de pouvoir, contre 33 pour les IATOS et 27 pour les extérieurs 2. 
Tableau 4 - Répartition du pouvoir, le président étant dans le conseil d'administration

\begin{tabular}{|c|cccccc|}
\hline Pouvoir & Professeurs & MCF & Étudiants & IATOS & Ext. 1 & Ext. 2 \\
\hline 35,71 & 2 & - & - & - & - & - \\
32,14 & 5 & - & - & - & - & - \\
30 & 8 & - & 1 & - & 7 & - \\
28,57 & 11 & 11 & - & - & - & - \\
26,67 & 10 & - & - & - & 5 & - \\
25 & - & 5 & - & - & - & - \\
23,33 & $\mathbf{4 2}$ & $\mathbf{3 6}$ & 8 & - & $\mathbf{3 4}$ & - \\
21,43 & - & 2 & - & - & 10 & - \\
20 & 8 & 17 & 6 & 1 & 11 & 2 \\
17,86 & - & - & 1 & - & 4 & - \\
16,67 & 10 & 22 & 24 & 5 & 16 & 7 \\
14,29 & - & - & 3 & - & 3 & 1 \\
13,33 & - & 1 & 12 & 8 & 2 & 7 \\
10,71 & - & - & 4 & 1 & 1 & 4 \\
10 & - & 2 & $\mathbf{2 7}$ & $\mathbf{4 8}$ & 3 & $\mathbf{4 8}$ \\
7,14 & - & - & 10 & 12 & - & 12 \\
6,67 & - & - & - & 7 & - & 8 \\
3,57 & - & - & - & 4 & - & 1 \\
3,33 & - & - & - & 7 & - & 5 \\
0 & - & - & - & 3 & - & 1 \\
\hline
\end{tabular}

\subsection{Le président est hors conseil}

Ajouter un membre au conseil d'administration, le président, en supposant que celui-ci est professeur des universités, redonne du pouvoir à ce groupe comme nous pouvons le voir dans le tableau 5 présenté en annexe. Ce tableau est bien sûr semblable au tableau 3 par construction mais il nous semble plus clair de présenter séparemment les deux cas. Une comparaison est possible pour mesurer l'impact d'un président élu hors conseil d'administration. Par exemple, si l'on compare les répartitions $[4,4,3,2,4,3] \mathrm{du}$ tableau 3 et $[5,4,3,2,4,3]$ du tableau 5 , la distribution de pouvoir est la même. Mais il est possible que le sort des étudiants, des IATOS et des extérieurs 2 s'améliore avec l'arrivée d'un nouveau professeur avec voix prépondérante. Si on ajoute à la répartition $[7,7,3,3,4,3]$ du tableau 3 , un professeur avec voix prépondérante (le président), on obtient la répartition $[8,7,3,3,4,3]$ du tableau 5 . Alors que le poids relatif des groupes étudiants, IATOS et extérieurs 2 diminue, leur pouvoir devient plus important $(10,71 \%$ contre $7,14 \%)$.

Grâce à l'arrivée d'un nouveau membre, le président, le groupe professeurs a un pouvoir renforcé. Bien entendu, les résultats seraient inversés avec les $M C F$ si le président en faisait partie. Si le mode est le même pour les $M C F$ et les étudiants (un pouvoir 
de 16,67\%), la distribution du pouvoir est favorable le plus souvent aux $M C F$. Encore une fois, les IATOS et extérieurs 2 sont ceux pour lesquels la distribution de pouvoir est la moins favorable. Ils ont moins de $10 \%$ du pouvoir dans respectivement 37 et 38 cas sur 96. Cela est vrai pour les étudiants dans seulement 9 cas.

Les répartitions de pouvoir par groupe sont synthétisées dans le tableau 6. Désormais, 24 valeurs différentes de pouvoir sont possibles (colonne $1 \mathrm{du}$ tableau 6), contre 20 dans le cas où le président était dans le conseil d'administration (tableau 4).

Tableau 6 - Répartition du pouvoir, le président étant hors du conseil d'administration

\begin{tabular}{|c|cccccc|}
\hline Pouvoir & Professeurs & MCF & Étudiants & IATOS & Ext. 1 & Ext. 2 \\
\hline 39,29 & 2 & - & - & - & - & - \\
35,71 & 11 & - & - & - & - & - \\
34,62 & 2 & - & - & - & - & - \\
33,33 & 2 & - & - & - & - & - \\
32,14 & 10 & - & - & - & - & - \\
30 & $\mathbf{2 4}$ & - & - & - & 1 & - \\
28,57 & 7 & 5 & - & - & 2 & - \\
26,92 & - & 2 & - & - & 2 & - \\
26,67 & 18 & - & - & - & 2 & - \\
25 & - & 10 & - & - & - & - \\
23,33 & 17 & 9 & 4 & - & $\mathbf{1 6}$ & - \\
21,43 & - & 13 & - & - & 5 & - \\
20 & 2 & 17 & 5 & - & $\mathbf{1 6}$ & - \\
17,86 & - & 2 & 1 & - & 9 & - \\
16,67 & 1 & $\mathbf{3 0}$ & $\mathbf{2 9}$ & 3 & 25 & 2 \\
14,29 & - & - & 11 & - & 11 & - \\
13,33 & - & 5 & 17 & 13 & 4 & 11 \\
10,71 & - & - & 11 & 6 & 3 & 8 \\
10 & - & 3 & 9 & $\mathbf{3 7}$ & - & $\mathbf{3 7}$ \\
7,14 & - & - & 7 & 18 & - & 18 \\
6,67 & - & - & - & 9 & - & 11 \\
3,85 & - & - & 2 & 2 & - & 2 \\
3,57 & - & - & - & 6 & - & 4 \\
3,33 & - & - & - & 2 & - & 3 \\
\hline
\end{tabular}

\section{Conclusion}

L'objet de cet article est d'étudier le pouvoir au sein des conseils d'administration des universités françaises, en vue de la loi relative aux libertés et responsabilités des universités (loi LRU). Cette loi a suscité bien des controverses et une préoccupation importante est l'importance du président, dans un conseil d'administration largement réduit, ainsi que le poids des différents groupes que nous avons appelés professeurs, $M C F$, 
étudiants, IATOS, extérieurs 1 et extérieurs 2. Par exemple, certains s'inquiètent du poids important des extérieurs et du poids faible des étudiants et/ou IATOS. La théorie des jeux coopératifs nous apprend, à travers la théorie des indices de pouvoir, que la seule distribution des représentants n'est pas suffisante pour déterminer le pouvoir des groupes. À partir de là, en utilisant ces outils, nous déterminons le pouvoir des différents groupes à l'aide de l'indice de Banzhaf, indice largement reconnu pour ses qualités normatives et la simplicité de sa présentation. Les résultats que nous obtenons sont parfois surprenants, voire paradoxaux. Par exemple, dans l'élection du président, le pouvoir des étudiants est important, tout du moins, il est le plus souvent aussi important que celui des professeurs et des $M C F$, alors même que leur poids relatif semble assez faible. Par contre, force est de reconnaître que le pouvoir des IATOS est faible, voire nul, dans bon nombre de cas. De plus, dans le conseil d'administration dans son ensemble, la situation des étudiants ne semble pas catastrophique et reste bien meilleure que celles des IATOS et extérieurs 2.

La portée de cet article se limite à l'analyse de pouvoir des groupes, en supposant que leurs membres votent de la même façon. Les groupes sont ceux définis par la loi. Cela signifie que l'analyse a un sens dans les cas où les décisions à prendre sont des décisions de groupes. Nous n'ignorons évidemment pas qu'à l'intérieur des groupes des dissensions existent, cela pouvant provenir des modes de scrutins pour l'élection des représentants. Au sein d'un même groupe, il est envisageable d'avoir des représentants issus de listes différentes et donc en possible désaccord. De la même manière, l'hypothèse d'indépendance entre les groupes peut être relachée. On pourrait supposer par exemple que la probabilité que les étudiants forment une coalition avec les professeurs est plus faible que la probabilité que les $M C F$ s'entendent avec ces mêmes professeurs. Toutes les situations sont alors imaginables et une analyse au cas par cas, université par université, serait nécessaire. Par exemple, nous avons supposé que le président est professeur des universités, mais il est possible que celui-ci soit élu grâce aux $M C F$ et non grâce aux professeurs. Il renforce alors le groupe $M C F$ et non professeurs, ce qui revient à ajouter un membre à voix prépondérante dans le groupe $M C F$. Pour en tenir compte, il faut alors supposer que le président est $M C F$ et notre analyse redevient tout à fait valable.

Dans cet article, nous présentons les pouvoirs dans le cadre de la réforme et aucune 
comparaison par rapport à l'existant, en terme de gain ou de perte de pouvoir n'est réalisée. Il n'est pas certain que cette comparaison soit pertinente étant donnée que la loi limite les marges de manoeuvres en encadrant les distributions de sièges possibles. Elle pourrait malgré tout donner peut-être des arguments à certains groupes en vue des négociations futures dans les conseils d'administration.

\section{Bibliographie}

Banzhaf. J.F, "Weighted voting doesn't work: A mathematical analysis", Rutgers Law Review, 19, p. 317 - 343, 1965.

Shapley. L.S et Shubik. M, "A method for evaluating the distribution of power in a committee system", American Political Science Review, 48, p. 787 - 792, 1954.

Straffin. P.D, "Power and stability in politics", Chapter 32, Aumann. R.J and Hart. S, eds, Handbook of Game Theory, vol 2, North-Holland, Amsterdam, The Netherlands, 1994.

Gouvernement français, "LOI n 2007-1199 du 10 août 2007 relative aux libertés et responsabilités des universités", J.O. n 185 du 11 août 2007 page 13468 , texte ${ }^{\circ}$ 2, 2007 :

http://www.legifrance.gouv.fr/WAspad/UnTexteDeJorf ?numjo=ESRX0757893L. 
Tableau 3 - président élu parmi le conseil d'administration

\begin{tabular}{|c|c|c|c|c|c|c|c|c|c|c|c|c|}
\hline \multicolumn{6}{|c|}{ Répartition de sièges } & \multirow[b]{2}{*}{$\bar{w}$} & \multicolumn{6}{|c|}{ Pouvoir } \\
\hline Pr. & $\mathrm{MCF}$ & Étu. & IATOS & Ext.1 & Ext.2 & & Pr. & MCF & Étu. & IATOS & Ext.1 & Ext.2 \\
\hline 4 & 4 & 3 & 2 & 4 & 3 & 20 & 26,67 & 20 & 13,33 & 6,67 & 20 & 13,33 \\
\hline 4 & 4 & 3 & 2 & 5 & 2 & 20 & 23,33 & 23,33 & 10 & 10 & 23,33 & 10 \\
\hline 4 & 4 & 3 & 2 & 5 & 3 & 21 & 20 & 20 & 13,33 & 6,67 & 26,67 & 13,33 \\
\hline 4 & 4 & 3 & 2 & 6 & 2 & 21 & 16,67 & 16,67 & 16,67 & 10 & 30 & 10 \\
\hline 4 & 4 & 4 & 2 & 4 & 3 & 21 & 20 & 20 & 20 & 0 & 20 & 20 \\
\hline 4 & 4 & 4 & 2 & 5 & 2 & 21 & 16,67 & 16,67 & 16,67 & 10 & 30 & 10 \\
\hline 4 & 4 & 3 & 3 & 4 & 3 & 21 & 23,33 & 23,33 & 10 & 10 & 23,33 & 10 \\
\hline 4 & 4 & 3 & 3 & 5 & 2 & 21 & 20 & 20 & 13,33 & 13,33 & 26,67 & 6,67 \\
\hline 5 & 5 & 3 & 2 & 4 & 3 & 22 & 26,67 & 20 & 13,33 & 6,67 & 20 & 13,33 \\
\hline 5 & 5 & 3 & 2 & 5 & 2 & 22 & 23,33 & 23,33 & 10 & 10 & 23,33 & 10 \\
\hline 4 & 4 & 4 & 2 & 5 & 3 & 22 & 23,33 & 16,67 & 16,67 & 3,33 & 23,33 & 16,67 \\
\hline 4 & 4 & 4 & 2 & 6 & 2 & 22 & 16,67 & 16,67 & 16,67 & 10 & 30 & 10 \\
\hline 4 & 4 & 3 & 3 & 5 & 3 & 22 & 23,33 & 23,33 & 10 & 10 & 23,33 & 10 \\
\hline 4 & 4 & 3 & 3 & 6 & 2 & 22 & 20 & 20 & 13,33 & 13,33 & 26,67 & 6,67 \\
\hline 4 & 4 & 5 & 2 & 4 & 3 & 22 & 23,33 & 16,67 & 23,33 & $\mathbf{3 , 3 3 3}$ & 16,67 & 16,67 \\
\hline 4 & 4 & 5 & 2 & 5 & 2 & 22 & 23,33 & 10 & 23,33 & 10 & 23,33 & 10 \\
\hline 4 & 4 & 4 & 3 & 4 & 3 & 22 & 30 & 16,67 & 16,67 & 10 & 16,67 & 10 \\
\hline 4 & 4 & 4 & 3 & 5 & 2 & 22 & 23,33 & 16,67 & 16,67 & 16,67 & 23,33 & 3,333 \\
\hline 5 & 5 & 3 & 2 & 5 & 3 & 23 & 23,33 & 23,33 & 10 & 10 & 23,33 & 10 \\
\hline 5 & 5 & 3 & 2 & 6 & 2 & 23 & 23,33 & 23,33 & 10 & 10 & 23,33 & 10 \\
\hline 5 & 5 & 4 & 2 & 4 & 3 & 23 & 23,33 & 23,33 & 16,67 & 3,33 & 16,67 & 16,67 \\
\hline 5 & 5 & 4 & 2 & 5 & 2 & 23 & 23,33 & 23,33 & 10 & 10 & 23,33 & 10 \\
\hline 5 & 5 & 3 & 3 & 4 & 3 & 23 & 23,33 & 23,33 & 10 & 10 & 23,33 & 10 \\
\hline 5 & 5 & 3 & 3 & 5 & 2 & 23 & 23,33 & 23,33 & 10 & 10 & 23,33 & 10 \\
\hline 4 & 4 & 5 & 2 & 5 & 3 & 23 & 16,67 & 16,67 & 23,33 & 3,33 & 23,33 & 16,67 \\
\hline 4 & 4 & 5 & 2 & 6 & 2 & 23 & 16,67 & 16,67 & 16,67 & 10 & 30 & 10 \\
\hline 4 & 4 & 4 & 3 & 5 & 3 & 23 & 16,67 & 16,67 & 16,67 & 10 & 30 & 10 \\
\hline 4 & 4 & 4 & 3 & 6 & 2 & 23 & 16,67 & 16,67 & 16,67 & 10 & 30 & 10 \\
\hline 4 & 4 & 5 & 3 & 4 & 3 & 23 & 16,67 & 16,67 & 30 & 10 & 16,67 & 10 \\
\hline 4 & 4 & 5 & 3 & 5 & 2 & 23 & 16,67 & 16,67 & 23,33 & 16,67 & 23,33 & 3,33 \\
\hline 6 & 6 & 3 & 2 & 4 & 3 & 24 & 32,14 & 25 & 10,71 & 3,57 & 17,86 & 10,71 \\
\hline 6 & 6 & 3 & 2 & 5 & 2 & 24 & 28,57 & 28,57 & 7,14 & 7,14 & 21,43 & 7,14 \\
\hline 5 & 5 & 4 & 2 & 5 & 3 & 24 & 26,67 & 20 & 13,33 & 6,67 & 20 & 13,33 \\
\hline 5 & 5 & 4 & 2 & 6 & 2 & 24 & 23,33 & 23,33 & 10 & 10 & 23,33 & 10 \\
\hline 5 & 5 & 3 & 3 & 5 & 3 & 24 & 23,33 & 23,33 & 10 & 10 & 23,33 & 10 \\
\hline 5 & 5 & 3 & 3 & 6 & 2 & 24 & 23,33 & 23,33 & 10 & 10 & 23,33 & 10 \\
\hline 5 & 5 & 5 & 2 & 4 & 3 & 24 & 26,67 & 20 & 20 & 6,67 & 13,33 & 13,33 \\
\hline 5 & 5 & 5 & 2 & 5 & 2 & 24 & 30 & 16,67 & 16,67 & 10 & 16,67 & 10 \\
\hline 5 & 5 & 4 & 3 & 4 & 3 & 24 & 30 & 16,67 & 16,67 & 10 & 16,67 & 10 \\
\hline 5 & 5 & 4 & 3 & 5 & 2 & 24 & 26,67 & 20 & 13,33 & 13,33 & 20 & 6,67 \\
\hline 4 & 4 & 5 & 3 & 5 & 3 & 24 & 23,33 & 10 & 23,33 & 10 & 23,33 & 10 \\
\hline 4 & 4 & 5 & 3 & 6 & 2 & 24 & 20 & 13,33 & 20 & 13,33 & 26,67 & 6,67 \\
\hline 6 & 6 & 3 & 2 & 5 & 3 & 25 & 23,33 & 23,33 & 10 & 10 & 23,33 & 10 \\
\hline 6 & 6 & 3 & 2 & 6 & 2 & 25 & 23,33 & 23,33 & 10 & 10 & 23,33 & 10 \\
\hline 6 & 6 & 4 & 2 & 4 & 3 & 25 & 23,33 & 23,33 & 16,67 & 3,33 & 16,67 & 16,67 \\
\hline 6 & 6 & 4 & 2 & 5 & 2 & 25 & 23,33 & 23,33 & 10 & 10 & 23,33 & 10 \\
\hline 6 & 6 & 3 & 3 & 4 & 3 & 25 & 23,33 & 23,33 & 10 & 10 & 23,33 & 10 \\
\hline 6 & 6 & 3 & 3 & 5 & 2 & 25 & 23,33 & 23,33 & 10 & 10 & 23,33 & 10 \\
\hline
\end{tabular}


Tableau 3 (suite) - président élu parmi le conseil d'administration

\begin{tabular}{|c|c|c|c|c|c|c|c|c|c|c|c|c|}
\hline \multicolumn{6}{|c|}{ Répartition de sièges } & \multirow[b]{2}{*}{$\bar{w}$} & \multicolumn{6}{|c|}{ Pouvoir } \\
\hline Pr. & MCF & Étu. & IATOS & Ext.1 & Ext.2 & & Pr. & $\mathrm{MCF}$ & Étu. & IATOS & Ext.1 & Ext.2 \\
\hline 5 & 5 & 5 & 2 & 5 & 3 & 25 & 20 & 20 & 20 & 0 & 20 & 20 \\
\hline 5 & 5 & 5 & 2 & 6 & 2 & 25 & 16,67 & 16,67 & 16,67 & 10 & 30 & 10 \\
\hline 5 & 5 & 4 & 3 & 5 & 3 & 25 & 23,33 & 23,33 & 10 & 10 & 23,33 & 10 \\
\hline 5 & 5 & 4 & 3 & 6 & 2 & 25 & 20 & 20 & 13,33 & 13,33 & 26,67 & 6,67 \\
\hline 5 & 5 & 5 & 3 & 4 & 3 & 25 & 23,33 & 23,33 & 23,33 & 10 & 10 & 10 \\
\hline 5 & 5 & 5 & 3 & 5 & 2 & 25 & 20 & 20 & 20 & 20 & 20 & 0 \\
\hline 7 & 7 & 3 & 2 & 4 & 3 & 26 & 32,14 & 25 & 10,71 & 3,57 & 17,86 & 10,71 \\
\hline 7 & 7 & 3 & 2 & 5 & 2 & 26 & 28,57 & 28,57 & 7,14 & 7,14 & 21,43 & 7,14 \\
\hline 6 & 6 & 4 & 2 & 5 & 3 & 26 & 26,67 & 20 & 13,33 & 6,67 & 20 & 13,33 \\
\hline 6 & 6 & 4 & 2 & 6 & 2 & 26 & 23,33 & 23,33 & 10 & 10 & 23,33 & 10 \\
\hline 6 & 6 & 3 & 3 & 5 & 3 & 26 & 23,33 & 23,33 & 10 & 10 & 23,33 & 10 \\
\hline 6 & 6 & 3 & 3 & 6 & 2 & 26 & 23,33 & 23,33 & 10 & 10 & 23,33 & 10 \\
\hline 6 & 6 & 5 & 2 & 4 & 3 & 26 & 26,67 & 20 & 20 & 6,67 & 13,33 & 13,33 \\
\hline 6 & 6 & 5 & 2 & 5 & 2 & 26 & 30 & 16,67 & 16,67 & 10 & 16,67 & 10 \\
\hline 6 & 6 & 4 & 3 & 4 & 3 & 26 & 30 & 16,67 & 16,67 & 10 & 16,67 & 10 \\
\hline 6 & 6 & 4 & 3 & 5 & 2 & 26 & 26,67 & 20 & 13,33 & 13,33 & 20 & 6,67 \\
\hline 5 & 5 & 5 & 3 & 5 & 3 & 26 & 30 & 16,67 & 16,67 & 10 & 16,67 & 10 \\
\hline 5 & 5 & 5 & 3 & 6 & 2 & 26 & 23,33 & 16,67 & 16,67 & 16,67 & 23,33 & 3,33 \\
\hline 7 & 7 & 3 & 2 & 5 & 3 & 27 & 28,57 & 28,57 & 7,14 & 7,14 & 21,43 & 7,14 \\
\hline 7 & 7 & 3 & 2 & 6 & 2 & 27 & 28,57 & 28,57 & 7,14 & 7,14 & 21,43 & 7,14 \\
\hline 7 & 7 & 4 & 2 & 4 & 3 & 27 & 28,57 & 28,57 & 14,29 & 0 & 14,29 & 14,29 \\
\hline 7 & 7 & 4 & 2 & 5 & 2 & 27 & 28,57 & 28,57 & 7,14 & 7,14 & 21,43 & 7,14 \\
\hline 7 & 7 & 3 & 3 & 4 & 3 & 27 & 28,57 & 28,57 & 7,14 & 7,14 & 21,43 & 7,14 \\
\hline 7 & 7 & 3 & 3 & 5 & 2 & 27 & 28,57 & 28,57 & 7,14 & 7,14 & 21,43 & 7,14 \\
\hline 6 & 6 & 5 & 2 & 5 & 3 & 27 & 23,33 & 23,33 & 16,67 & 3,33 & 16,67 & 16,67 \\
\hline 6 & 6 & 5 & 2 & 6 & 2 & 27 & 23,33 & 23,33 & 10 & 10 & 23,33 & 10 \\
\hline 6 & 6 & 4 & 3 & 5 & 3 & 27 & 23,33 & 23,33 & 10 & 10 & 23,33 & 10 \\
\hline 6 & 6 & 4 & 3 & 6 & 2 & 27 & 23,33 & 23,33 & 10 & 10 & 23,33 & 10 \\
\hline 6 & 6 & 5 & 3 & 4 & 3 & 27 & 23,33 & 23,33 & 23,33 & 10 & 10 & 10 \\
\hline 6 & 6 & 5 & 3 & 5 & 2 & 27 & 23,33 & 23,33 & 16,67 & 16,67 & 16,67 & 3,33 \\
\hline 7 & 7 & 4 & 2 & 5 & 3 & 28 & 32,14 & 25 & 10,71 & 3,57 & 17,86 & 10,71 \\
\hline 7 & 7 & 4 & 2 & 6 & 2 & 28 & 28,57 & 28,57 & 7,14 & 7,14 & 21,43 & 7,14 \\
\hline 7 & 7 & 3 & 3 & 5 & 3 & 28 & 28,57 & 28,57 & 7,14 & 7,14 & 21,43 & 7,14 \\
\hline 7 & 7 & 3 & 3 & 6 & 2 & 28 & 28,57 & 28,57 & 7,14 & 7,14 & 21,43 & 7,14 \\
\hline 7 & 7 & 5 & 2 & 4 & 3 & 28 & 32,14 & 25 & 17,86 & 3,57 & 10,71 & 10,71 \\
\hline 7 & 7 & 5 & 2 & 5 & 2 & 28 & 35,71 & 21,43 & 14,29 & 7,14 & 14,29 & 7,14 \\
\hline 7 & 7 & 4 & 3 & 4 & 3 & 28 & 35,71 & 21,43 & 14,29 & 7,14 & 14,29 & 7,14 \\
\hline 7 & 7 & 4 & 3 & 5 & 2 & 28 & 32,14 & 25 & 10,71 & 10,71 & 17,86 & 3,57 \\
\hline 6 & 6 & 5 & 3 & 5 & 3 & 28 & 30 & 16,67 & 16,67 & 10 & 16,67 & 10 \\
\hline 6 & 6 & 5 & 3 & 6 & 2 & 28 & 26,67 & 20 & 13,33 & 13,33 & 20 & 6,67 \\
\hline 7 & 7 & 5 & 2 & 5 & 3 & 29 & 23,33 & 23,33 & 16,67 & 3,33 & 16,67 & 16,67 \\
\hline 7 & 7 & 5 & 2 & 6 & 2 & 29 & 23,33 & 23,33 & 10 & 10 & 23,33 & 10 \\
\hline 7 & 7 & 4 & 3 & 5 & 3 & 29 & 23,33 & 23,33 & 10 & 10 & 23,33 & 10 \\
\hline 7 & 7 & 4 & 3 & 6 & 2 & 29 & 23,33 & 23,33 & 10 & 10 & 23,33 & 10 \\
\hline 7 & 7 & 5 & 3 & 4 & 3 & 29 & 23,33 & 23,33 & 23,33 & 10 & 10 & 10 \\
\hline 7 & 7 & 5 & 3 & 5 & 2 & 29 & 23,33 & 23,33 & 16,67 & 16,67 & 16,67 & 3,33 \\
\hline 7 & 7 & 5 & 3 & 5 & 3 & 30 & 30 & 16,67 & 16,67 & 10 & 16,67 & 10 \\
\hline 7 & 7 & 5 & 3 & 6 & 2 & 30 & 26,67 & 20 & 13,33 & 13,33 & 20 & 6,67 \\
\hline
\end{tabular}


Tableau 5 - président élu hors conseil d'administration

\begin{tabular}{|c|c|c|c|c|c|c|c|c|c|c|c|c|}
\hline \multicolumn{6}{|c|}{ Répartition de sièges } & \multirow[b]{2}{*}{$\bar{w}$} & \multicolumn{6}{|c|}{ Pouvoir } \\
\hline Pr. & $\mathrm{MCF}$ & Étu. & IATOS & Ext.1 & Ext.2 & & Pr. & $\mathrm{MCF}$ & Étu. & IATOS & Ext.1 & Ext.2 \\
\hline 5 & 4 & 3 & 2 & 4 & 3 & 21 & 26,67 & 20 & 13,33 & 6,67 & 20 & 13,33 \\
\hline 5 & 4 & 3 & 2 & 5 & 2 & 21 & 23,33 & 23,33 & 10 & 10 & 23,33 & 10 \\
\hline 5 & 4 & 3 & 2 & 5 & 3 & 22 & 26,67 & 20 & 13,33 & 6,67 & 20 & 13,33 \\
\hline 5 & 4 & 3 & 2 & 6 & 2 & 22 & 28,57 & 21,43 & 7,14 & 7,14 & 28,57 & 7,14 \\
\hline 5 & 4 & 4 & 2 & 4 & 3 & 22 & 30 & 16,67 & 16,67 & 10 & 16,67 & 10 \\
\hline 5 & 4 & 4 & 2 & 5 & 2 & 22 & 30 & 16,67 & 16,67 & 10 & 16,67 & 10 \\
\hline 5 & 4 & 3 & 3 & 4 & 3 & 22 & 33,33 & 13,33 & 13,33 & 13,33 & 13,33 & 13,33 \\
\hline 5 & 4 & 3 & 3 & 5 & 2 & 22 & 26,67 & 20 & 13,33 & 13,33 & 20 & 6,67 \\
\hline 6 & 5 & 3 & 2 & 4 & 3 & 23 & 26,67 & 20 & 13,33 & 6,67 & 20 & 13,33 \\
\hline 6 & 5 & 3 & 2 & 5 & 2 & 23 & 23,33 & 23,33 & 10 & 10 & 23,33 & 10 \\
\hline 5 & 4 & 4 & 2 & 5 & 3 & 23 & 23,33 & 16,67 & 16,67 & 3,33 & 23,33 & 16,67 \\
\hline 5 & 4 & 4 & 2 & 6 & 2 & 23 & 16,67 & 16,67 & 16,67 & 10 & 30 & 10 \\
\hline 5 & 4 & 3 & 3 & 5 & 3 & 23 & 23,33 & 23,33 & 10 & 10 & 23,33 & 10 \\
\hline 5 & 4 & 3 & 3 & 6 & 2 & 23 & 20 & 20 & 13,33 & 13,33 & 26,67 & 6,67 \\
\hline 5 & 4 & 5 & 2 & 4 & 3 & 23 & 23,33 & 16,67 & 23,33 & 3,33 & 16,67 & 16,67 \\
\hline 5 & 4 & 5 & 2 & 5 & 2 & 23 & 23,33 & 10 & 23,33 & 10 & 23,33 & 10 \\
\hline 5 & 4 & 4 & 3 & 4 & 3 & 23 & 30 & 16,67 & 16,67 & 10 & 16,67 & 10 \\
\hline 5 & 4 & 4 & 3 & 5 & 2 & 23 & 23,33 & 16,67 & 16,67 & 16,67 & 23,33 & 3,33 \\
\hline 6 & 5 & 3 & 2 & 5 & 3 & 24 & 26,67 & 20 & 13,33 & 6,67 & 20 & 13,33 \\
\hline 6 & 5 & 3 & 2 & 6 & 2 & 24 & 28,57 & 21,43 & 7,14 & 7,14 & 28,57 & 7,14 \\
\hline 6 & 5 & 4 & 2 & 4 & 3 & 24 & 30 & 16,67 & 16,67 & 10 & 16,67 & 10 \\
\hline 6 & 5 & 4 & 2 & 5 & 2 & 24 & 30 & 16,67 & 16,67 & 10 & 16,67 & 10 \\
\hline 6 & 5 & 3 & 3 & 4 & 3 & 24 & 33,33 & 13,33 & 13,33 & 13,33 & 13,33 & 13,33 \\
\hline 6 & 5 & 3 & 3 & 5 & 2 & 24 & 26,67 & 20 & 13,33 & 13,33 & 20 & 6,67 \\
\hline 5 & 4 & 5 & 2 & 5 & 3 & 24 & 26,67 & 13,33 & 20 & 6,67 & 20 & 13,33 \\
\hline 5 & 4 & 5 & 2 & 6 & 2 & 24 & 23,33 & 10 & 23,33 & 10 & 23,33 & 10 \\
\hline 5 & 4 & 4 & 3 & 5 & 3 & 24 & 30 & 16,67 & 16,67 & 10 & 16,67 & 10 \\
\hline 5 & 4 & 4 & 3 & 6 & 2 & 24 & 23,33 & 16,67 & 16,67 & 16,67 & 23,33 & 3,33 \\
\hline 5 & 4 & 5 & 3 & 4 & 3 & 24 & 30 & 16,67 & 16,67 & 10 & 16,67 & 10 \\
\hline 5 & 4 & 5 & 3 & 5 & 2 & 24 & 26,67 & 13,33 & 20 & 13,33 & 20 & 6,67 \\
\hline 7 & 6 & 3 & 2 & 4 & 3 & 25 & 32,14 & 25 & 10,71 & 3,57 & 17,86 & 10,71 \\
\hline 7 & 6 & 3 & 2 & 5 & 2 & 25 & 28,57 & 28,57 & 7,14 & 7,14 & 21,43 & 7,14 \\
\hline 6 & 5 & 4 & 2 & 5 & 3 & 25 & 26,67 & 20 & 13,33 & 6,67 & 20 & 13,33 \\
\hline 6 & 5 & 4 & 2 & 6 & 2 & 25 & 23,33 & 23,33 & 10 & 10 & 23,33 & 10 \\
\hline 6 & 5 & 3 & 3 & 5 & 3 & 25 & 23,33 & 23,33 & 10 & 10 & 23,33 & 10 \\
\hline 6 & 5 & 3 & 3 & 6 & 2 & 25 & 23,33 & 23,33 & 10 & 10 & 23,33 & 10 \\
\hline 6 & 5 & 5 & 2 & 4 & 3 & 25 & 26,67 & 20 & 20 & 6,67 & 13,33 & 13,33 \\
\hline 6 & 5 & 5 & 2 & 5 & 2 & 25 & 30 & 16,67 & 16,67 & 10 & 16,67 & 10 \\
\hline 6 & 5 & 4 & 3 & 4 & 3 & 25 & 30 & 16,67 & 16,67 & 10 & 16,67 & 10 \\
\hline 6 & 5 & 4 & 3 & 5 & 2 & 25 & 26,67 & 20 & 13,33 & 13,33 & 20 & 6,67 \\
\hline 5 & 4 & 5 & 3 & 5 & 3 & 25 & 23,33 & 10 & 23,33 & 10 & 23,33 & 10 \\
\hline 5 & 4 & 5 & 3 & 6 & 2 & 25 & 20 & 13,33 & 20 & 13,33 & 26,67 & 6,67 \\
\hline 7 & 6 & 3 & 2 & 5 & 3 & 26 & 32,14 & 25 & 10,71 & 3,57 & 17,86 & 10,71 \\
\hline 7 & 6 & 3 & 2 & 6 & 2 & 26 & 34,62 & 26,92 & 3,85 & 3,85 & 26,92 & 3,85 \\
\hline 7 & 6 & 4 & 2 & 4 & 3 & 26 & 35,71 & 21,43 & 14,29 & 7,14 & 14,29 & 7,14 \\
\hline 7 & 6 & 4 & 2 & 5 & 2 & 26 & 35,71 & 21,43 & 14,29 & 7,14 & 14,29 & 7,14 \\
\hline 7 & 6 & 3 & 3 & 4 & 3 & 26 & 39,29 & 17,86 & 10,71 & 10,71 & 10,71 & 10,71 \\
\hline 7 & 6 & 3 & 3 & 5 & 2 & 26 & 32,14 & 25 & 10,71 & 10,71 & 17,86 & 3,57 \\
\hline
\end{tabular}


Tableau 5 (suite) - président élu hors conseil d'administration

\begin{tabular}{|c|c|c|c|c|c|c|c|c|c|c|c|c|}
\hline \multirow[b]{2}{*}{ Pr. } & \multicolumn{5}{|c|}{ Répartition de sièges } & \multirow[b]{2}{*}{$\bar{w}$} & \multicolumn{6}{|c|}{ Pouvoir } \\
\hline & $\mathrm{MCF}$ & Étu. & IATOS & Ext.1 & Ext.2 & & Pr. & $\mathrm{MCF}$ & Étu. & IATOS & Ext.1 & Ext.2 \\
\hline 6 & 5 & 5 & 2 & 5 & 3 & 26 & 30 & 16,67 & 16,67 & 10 & 16,67 & 10 \\
\hline 6 & 5 & 5 & 2 & 6 & 2 & 26 & 30 & 16,67 & 16,67 & 10 & 16,67 & 10 \\
\hline 6 & 5 & 4 & 3 & 5 & 3 & 26 & 30 & 16,67 & 16,67 & 10 & 16,67 & 10 \\
\hline 6 & 5 & 4 & 3 & 6 & 2 & 26 & 26,67 & 20 & 13,33 & 13,33 & 20 & 6,67 \\
\hline 6 & 5 & 5 & 3 & 4 & 3 & 26 & 30 & 16,67 & 16,67 & 10 & 16,67 & 10 \\
\hline 6 & 5 & 5 & 3 & 5 & 2 & 26 & 30 & 16,67 & 16,67 & 10 & 16,67 & 10 \\
\hline 8 & 7 & 3 & 2 & 4 & 3 & 27 & 32,14 & 25 & 10,71 & 3,57 & 17,86 & 10,71 \\
\hline 8 & 7 & 3 & 2 & 5 & 2 & 27 & 28,57 & 28,57 & 7,14 & 7,14 & 21,43 & 7,14 \\
\hline 7 & 6 & 4 & 2 & 5 & 3 & 27 & 26,67 & 20 & 13,33 & 6,67 & 20 & 13,33 \\
\hline 7 & 6 & 4 & 2 & 6 & 2 & 27 & 23,33 & 23,33 & 10 & 10 & 23,33 & 10 \\
\hline 7 & 6 & 3 & 3 & 5 & 3 & 27 & 23,33 & 23,33 & 10 & 10 & 23,33 & 10 \\
\hline 7 & 6 & 3 & 3 & 6 & 2 & 27 & 23,33 & 23,33 & 10 & 10 & 23,33 & 10 \\
\hline 7 & 6 & 5 & 2 & 4 & 3 & 27 & 26,67 & 20 & 20 & 6,67 & 13,33 & 13,33 \\
\hline 7 & 6 & 5 & 2 & 5 & 2 & 27 & 30 & 16,67 & 16,67 & 10 & 16,67 & 10 \\
\hline 7 & 6 & 4 & 3 & 4 & 3 & 27 & 30 & 16,67 & 16,67 & 10 & 16,67 & 10 \\
\hline 7 & 6 & 4 & 3 & 5 & 2 & 27 & 26,67 & 20 & 13,33 & 13,33 & 20 & 6,67 \\
\hline 6 & 5 & 5 & 3 & 5 & 3 & 27 & 30 & 16,67 & 16,67 & 10 & 16,67 & 10 \\
\hline 6 & 5 & 5 & 3 & 6 & 2 & 27 & 23,33 & 16,67 & 16,67 & 16,67 & 23,33 & 3,33 \\
\hline 8 & 7 & 3 & 2 & 5 & 3 & 28 & 32,14 & 25 & 10,71 & 3,57 & 17,86 & 10,71 \\
\hline 8 & 7 & 3 & 2 & 6 & 2 & 28 & 34,62 & 26,92 & 3,85 & 3,85 & 26,92 & 3,85 \\
\hline 8 & 7 & 4 & 2 & 4 & 3 & 28 & 35,71 & 21,43 & 14,29 & 7,14 & 14,29 & 7,14 \\
\hline 8 & 7 & 4 & 2 & 5 & 2 & 28 & 35,71 & 21,43 & 14,29 & 7,14 & 14,29 & 7,14 \\
\hline 8 & 7 & 3 & 3 & 4 & 3 & 28 & 39,29 & 17,86 & 10,71 & 10,71 & 10,71 & 10,71 \\
\hline 8 & 7 & 3 & 3 & 5 & 2 & 28 & 32,14 & 25 & 10,71 & 10,71 & 17,86 & 3,57 \\
\hline 7 & 6 & 5 & 2 & 5 & 3 & 28 & 30 & 16,67 & 16,67 & 10 & 16,67 & 10 \\
\hline 7 & 6 & 5 & 2 & 6 & 2 & 28 & 30 & 16,67 & 16,67 & 10 & 16,67 & 10 \\
\hline 7 & 6 & 4 & 3 & 5 & 3 & 28 & 30 & 16,67 & 16,67 & 10 & 16,67 & 10 \\
\hline 7 & 6 & 4 & 3 & 6 & 2 & 28 & 26,67 & 20 & 13,33 & 13,33 & 20 & 6,67 \\
\hline 7 & 6 & 5 & 3 & 4 & 3 & 28 & 30 & 16,67 & 16,67 & 10 & 16,67 & 10 \\
\hline 7 & 6 & 5 & 3 & 5 & 2 & 28 & 30 & 16,67 & 16,67 & 10 & 16,67 & 10 \\
\hline 8 & 7 & 4 & 2 & 5 & 3 & 29 & 32,14 & 25 & 10,71 & 3,57 & 17,86 & 10,71 \\
\hline 8 & 7 & 4 & 2 & 6 & 2 & 29 & 28,57 & 28,57 & 7,14 & 7,14 & 21,43 & 7,14 \\
\hline 8 & 7 & 3 & 3 & 5 & 3 & 29 & 28,57 & 28,57 & 7,14 & 7,14 & 21,43 & 7,14 \\
\hline 8 & 7 & 3 & 3 & 6 & 2 & 29 & 28,57 & 28,57 & 7,14 & 7,14 & 21,43 & 7,14 \\
\hline 8 & 7 & 5 & 2 & 4 & 3 & 29 & 32,14 & 25 & 17,86 & 3,57 & 10,71 & 10,71 \\
\hline 8 & 7 & 5 & 2 & 5 & 2 & 29 & 35,71 & 21,43 & 14,29 & 7,14 & 14,29 & 7,14 \\
\hline 8 & 7 & 4 & 3 & 4 & 3 & 29 & 35,71 & 21,43 & 14,29 & 7,14 & 14,29 & 7,14 \\
\hline 8 & 7 & 4 & 3 & 5 & 2 & 29 & 32,14 & 25 & 10,71 & 10,71 & 17,86 & 3,57 \\
\hline 7 & 6 & 5 & 3 & 5 & 3 & 29 & 30 & 16,67 & 16,67 & 10 & 16,67 & 10 \\
\hline 7 & 6 & 5 & 3 & 6 & 2 & 29 & 26,67 & 20 & 13,33 & 13,33 & 20 & 6,67 \\
\hline 8 & 7 & 5 & 2 & 5 & 3 & 30 & 35,71 & 21,43 & 14,29 & 7,14 & 14,29 & 7,14 \\
\hline 8 & 7 & 5 & 2 & 6 & 2 & 30 & 35,71 & 21,43 & 14,29 & 7,14 & 14,29 & 7,14 \\
\hline 8 & 7 & 4 & 3 & 5 & 3 & 30 & 35,71 & 21,43 & 14,29 & 7,14 & 14,29 & 7,14 \\
\hline 8 & 7 & 4 & 3 & 6 & 2 & 30 & 32,14 & 25 & 10,71 & 10,71 & 17,86 & 3,57 \\
\hline 8 & 7 & 5 & 3 & 4 & 3 & 30 & 35,71 & 21,43 & 14,29 & 7,14 & 14,29 & 7,14 \\
\hline 8 & 7 & 5 & 3 & 5 & 2 & 30 & 35,71 & 21,43 & 14,29 & 7,14 & 14,29 & 7,14 \\
\hline 8 & 7 & 5 & 3 & 5 & 3 & 31 & 30 & 16,67 & 16,67 & 10 & 16,67 & 10 \\
\hline 8 & 7 & 5 & 3 & 6 & 2 & 31 & 26,67 & 20 & 13,33 & 13,33 & 20 & 6,67 \\
\hline
\end{tabular}

